\title{
The index of analytic vector fields and Newton polyhedra
}

\author{
by \\ Carles Bivià-Ausina (València)
}

\begin{abstract}
We prove that if $f:\left(\mathbb{R}^{n}, 0\right) \rightarrow\left(\mathbb{R}^{n}, 0\right)$ is an analytic map germ such that $f^{-1}(0)=\{0\}$ and $f$ satisfies a certain non-degeneracy condition with respect to a Newton polyhedron $\Gamma_{+} \subseteq \mathbb{R}^{n}$, then the index of $f$ only depends on the principal parts of $f$ with respect to the compact faces of $\Gamma_{+}$. In particular, we obtain a known result on the index of semi-weighted-homogeneous map germs. We also discuss non-degenerate vector fields in the sense of Khovanskiı and special applications of our results to planar analytic vector fields.
\end{abstract}

1. Introduction. Let $f:\left(\mathbb{R}^{n}, 0\right) \rightarrow\left(\mathbb{R}^{n}, 0\right)$ be a continuous map germ such that 0 is isolated in $f^{-1}(0)$. Then the index or mapping degree of $f$, denoted by $\operatorname{ind}_{0}(f)$, is a well known topological invariant of $f$ (see [7], [12] or [18] for the definition and properties of this invariant). In this paper we give a qualitative result on the index of analytic map germs $f:\left(\mathbb{R}^{n}, 0\right) \rightarrow\left(\mathbb{R}^{n}, 0\right)$ which are strongly adapted to a given Newton polyhedron $\Gamma_{+}$in $\mathbb{R}^{n}$ (see Definition 2.3). This notion was introduced by the author in [2] in order to give estimates for Łojasiewicz exponents of analytic functions $\left(\mathbb{R}^{n}, 0\right) \rightarrow$ $(\mathbb{R}, 0)$ with an isolated singularity at the origin.

The class of maps strongly adapted to a given Newton polyhedron $\Gamma_{+}$ contains the class of non-degenerate maps on $\Gamma_{+}$, a notion defined in [3] using the Newton filtration induced by $\Gamma_{+}$, thus generalizing semi-weightedhomogeneous maps. In the present paper we generalize the main result of Cima-Gasull-Torregrosa [6] on the index of a semi-weighted-homogeneous map germ $\left(\mathbb{R}^{n}, 0\right) \rightarrow\left(\mathbb{R}^{n}, 0\right)$; we shall state this result after some preliminary definitions.

Let $x_{1}, \ldots, x_{n}$ be a coordinate system in $\mathbb{R}^{n}$ and let $\mathbf{w}=\left(w_{1}, \ldots, w_{n}\right)$, where each $w_{i}$ is a positive integer. Given a monomial $x^{k}=x_{1}^{k_{1}} \ldots x_{n}^{k_{n}}, k_{i} \geq 0$,

2000 Mathematics Subject Classification: Primary 32S05; Secondary 57R45. hedra.

Key words and phrases: index of a vector field, real-analytic functions, Newton poly-

Work supported by DGICYT Grant BFM2000-1110. 
and $d>0$, we say that $x^{k}$ has $\mathbf{w}$-degree $d$ if $w_{1} k_{1}+\ldots+w_{n} k_{n}=d$. If $d_{1}, \ldots, d_{p}$ are positive numbers, a polynomial map $g=\left(g_{1}, \ldots, g_{p}\right)$ : $\left(\mathbb{R}^{n}, 0\right) \rightarrow\left(\mathbb{R}^{p}, 0\right)$ is said to be weighted homogeneous of weights $w_{1}, \ldots, w_{n}$ and degrees $d_{1}, \ldots, d_{p}$ if each monomial $x^{k}$ appearing in the expression of $g_{i}$ has w-degree $d_{i}$ for all $i=1, \ldots, p$.

Suppose that $g:\left(\mathbb{R}^{n}, 0\right) \rightarrow\left(\mathbb{R}^{p}, 0\right)$ is a weighted homogeneous map germ of weights $w_{1}, \ldots, w_{n}$ and degrees $d_{1}, \ldots, d_{p}$ such that $g^{-1}(0)=\{0\}$. If $G=\left(G_{1}, \ldots, G_{p}\right):\left(\mathbb{R}^{n}, 0\right) \rightarrow\left(\mathbb{R}^{p}, 0\right)$ is an analytic map germ such that every monomial $x^{k}$ appearing in the Taylor expansion of $G_{i}$ has $\mathbf{w}$-degree $>d_{i}$ for all $i=1, \ldots, p$, then the map $g+G$ is said to be semi-weightedhomogeneous of weights $w_{1}, \ldots, w_{n}$ and degrees $d_{1}, \ldots, d_{p}$. In this case, the map $g$ is called the weighted homogeneous part of $g+G$ or the principal part of $g+G$ with respect to the weights $w_{1}, \ldots, w_{n}$.

The motivation of our work is the following result.

Theorem $1.1([6])$. Let $f=g+G:\left(\mathbb{R}^{n}, 0\right) \rightarrow\left(\mathbb{R}^{n}, 0\right)$ be a semiweighted-homogeneous map germ, where $g$ is the weighted homogeneous part of $f$. Then $(g+G)^{-1}(0)=\{0\}$ and $\operatorname{ind}_{0}(g)=\operatorname{ind}_{0}(g+G)$.

We give a generalization of the above theorem by considering map germs strongly adapted to a given Newton polyhedron instead of semi-weightedhomogeneous map germs (see Theorem 2.8). The result thus obtained is intended to be useful in differential equations and differential geometry. We also study, in Section 4, how systems strongly adapted to a given Newton polyhedron are related to strongly non-degenerate systems, a notion inspired by Khovanski1's definition of non-degenerate complete intersection (see Definition 4.6). A corollary of our main result in particular applies to planar analytic vector fields (see Corollary 4.8). Further works analysing topological information supplied by certain principal parts of planar vector fields are due to Brunella-Miari [5] and Županović [21].

2. Result. We set $\mathbb{R}_{+}=\{x \in \mathbb{R}: x \geq 0\}, \mathbb{Q}_{+}=\mathbb{Q} \cap \mathbb{R}_{+}$and $\mathbb{Z}_{+}=$ $\mathbb{Z} \cap \mathbb{R}_{+}$.

Definition 2.1. We say that $\Gamma_{+} \subseteq \mathbb{R}_{+}^{n}$ is a Newton polyhedron if there exists some set $A \subseteq \mathbb{Q}_{+}^{n}$ such that $\Gamma_{+}$is the convex hull in $\mathbb{R}_{+}^{n}$ of $\{k+v$ : $\left.k \in A, v \in \mathbb{R}_{+}^{n}\right\}$. Then $\Gamma_{+}$is also called the Newton polyhedron determined by $A$.

Let $\mathcal{A}_{n}$ be the local ring of analytic function germs $g:\left(\mathbb{R}^{n}, 0\right) \rightarrow \mathbb{R}$ and let $m_{n}$ be its maximal ideal. If $g=\sum_{k} a_{k} x^{k}$ is the Taylor expansion of a germ $g \in \mathcal{A}_{n}$, the support of $g$ is the $\operatorname{set} \operatorname{supp}(g)=\left\{k \in \mathbb{Z}_{+}^{n}: a_{k} \neq 0\right\}$. If $g=0$, then $\operatorname{supp}(g)=\emptyset$. 
Given $g \in \mathcal{A}_{n}$, the Newton polyhedron of $g$, denoted by $\Gamma_{+}(g)$, is the Newton polyhedron determined by $\operatorname{supp}(g)$. If $g=0$, then $\Gamma_{+}(g)=\emptyset$. If $S=\left\{g_{1}, \ldots, g_{s}\right\} \subseteq \mathcal{A}_{n}$, the Newton polyhedron of $S, \Gamma_{+}(S)$, is the convex hull of $\Gamma_{+}\left(g_{1}\right) \cup \ldots \cup \Gamma_{+}\left(g_{s}\right)$. If $I$ is an ideal of $\mathcal{A}_{n}$ and $S$ is a generating system of $I$, we define the Newton polyhedron of $I$ as $\Gamma_{+}(I)=\Gamma_{+}(S)$, where $S$ is any generating system of $I$. It is immediate to check that this definition does not depend on the chosen generating system of $I$.

We denote by $\langle u, v\rangle$ the usual scalar product of two vectors $u, v \in \mathbb{R}^{n}$. If $v \in \mathbb{R}_{+}^{n} \backslash\{0\}$ and $\Gamma_{+}$is a given Newton polyhedron, we define $\ell\left(v, \Gamma_{+}\right)=$ $\min \left\{\langle k, v\rangle: k \in \Gamma_{+}\right\}$and $\Delta\left(v, \Gamma_{+}\right)=\left\{k \in \Gamma_{+}:\langle k, v\rangle=\ell\left(v, \Gamma_{+}\right)\right\}$. Every set of the form $\Delta\left(v, \Gamma_{+}\right)$is called a face of $\Gamma_{+}$; in this case we say that $v$ supports $\Delta\left(v, \Gamma_{+}\right)$. The dimension of a face $\Delta$ of $\Gamma_{+}$is the dimension of the smallest affine subspace containing $\Delta$. We denote by $\Gamma$ the union of all compact faces of $\Gamma_{+}$. If $S \subseteq \mathcal{A}_{n}$, then $\Gamma(S)$ is defined analogously.

A vector $v \in \mathbb{Z}_{+}^{n} \backslash\{0\}$ is said to be primitive if it is the vector with smallest length among all the vectors in the line determined by $v$, that is, $v$ has mutually prime integer coordinates. Every face of dimension $n-1$ of a Newton polyhedron $\Gamma_{+} \subseteq \mathbb{R}^{n}$ is supported by a unique primitive vector. We denote the set of primitive vectors supporting some face of $\Gamma_{+}$of dimension $n-1$ by $\mathcal{F}\left(\Gamma_{+}\right)$. We write $\mathcal{F}_{\mathrm{c}}\left(\Gamma_{+}\right)$to denote the subset of $\mathcal{F}\left(\Gamma_{+}\right)$given by those vectors supporting some compact face of $\Gamma_{+}$of dimension $n-1$. We observe that $\mathcal{F}_{\mathrm{c}}\left(\Gamma_{+}\right) \subseteq\left(\mathbb{R}_{+} \backslash\{0\}\right)^{n}$. If $S \subseteq \mathcal{A}_{n}$ then we denote $\mathcal{F}\left(\Gamma_{+}(S)\right)$ and $\mathcal{F}_{\mathrm{c}}\left(\Gamma_{+}(S)\right)$ by $\mathcal{F}(S)$ and $\mathcal{F}_{\mathrm{c}}(S)$, respectively.

We observe that any Newton polyhedron $\Gamma_{+} \subseteq \mathbb{R}_{+}^{n}$ can be expressed as

$$
\Gamma_{+}=\left\{k \in \mathbb{R}_{+}^{n}:\langle k, v\rangle \geq \ell\left(v, \Gamma_{+}\right) \text {for all } v \in \mathcal{F}\left(\Gamma_{+}\right)\right\} .
$$

If $\Gamma_{+} \subseteq \mathbb{R}^{n}$ is a Newton polyhedron intersecting each coordinate axis, then it is easy to check that $\mathcal{F}\left(\Gamma_{+}\right)=\mathcal{F}_{\mathrm{c}}\left(\Gamma_{+}\right) \cup\left\{e_{1}, \ldots, e_{n}\right\}$, where $e_{1}, \ldots, e_{n}$ is the canonical basis in $\mathbb{R}^{n}$.

Given $v \in \mathbb{R}_{+}^{n} \backslash\{0\}$ and $g \in \mathcal{A}_{n}, g \neq 0$, we set $\ell(v, g)=\ell\left(v, \Gamma_{+}(g)\right)$ and $\Delta(v, g)=\Delta\left(v, \Gamma_{+}(g)\right)$. If $g=0$, then we define $\ell(v, g)=0$ and $\Delta(v, g)=\emptyset$.

Let $\mathbf{w}=\left(w_{1}, \ldots, w_{n}\right)$, where each $w_{i}$ is a positive integer. Then we can consider the Newton polyhedron $\Gamma_{+}$determined by the points $\left\{w_{i}^{-1} e_{i}: i=\right.$ $1, \ldots, n\}$. We have $\mathcal{F}_{\mathrm{c}}\left(\Gamma_{+}\right)=\{\mathbf{w}\}$ and $\mathcal{F}\left(\Gamma_{+}\right)=\left\{\mathbf{w}, e_{1}, \ldots, e_{n}\right\}$. If $g \in \mathcal{A}_{n}$, then $\ell(\mathbf{w}, g)$ is the minimum of the $\mathbf{w}$-degrees of the monomials $x^{k}$ such that $k \in \operatorname{supp}(g)$.

Definition 2.2. If $g=\sum_{k} a_{k} x^{k} \in \mathcal{A}_{n}, g \neq 0$, and $v \in \mathbb{R}_{+}^{n}$, we define the principal part of $g$ with respect to $v$ as the germ $p_{v}(g) \in \mathcal{A}_{n}$ given by the sum of those $a_{k} x^{k}$ such that $k \in \Delta(v, g)$. If $g=0$, then we set $p_{v}(g)=0$.

Let $\Gamma_{+} \subseteq \mathbb{R}^{n}$ be a Newton polyhedron. If $J \subseteq \mathcal{F}\left(\Gamma_{+}\right)$and $g=\sum_{k} a_{k} x^{k}$ $\in \mathcal{A}_{n}$, then we define the principal part of $g$ with respect to $J$ as the germ 
$p_{J}(g) \in \mathcal{A}_{n}$ given by the sum of those $a_{k} x^{k}$ such that $k \in \bigcap_{v \in J} \Delta(v, g)$. The set $\bigcap_{v \in J} \Delta(v, g)$ could be empty; in this case, we set $p_{J}(g)=0$.

Definition $2.3([2])$. Let $S=\left\{g_{1}, \ldots, g_{s}\right\} \subseteq \mathcal{A}_{n}$ and let $\Gamma_{+} \subseteq \mathbb{R}^{n}$ be a Newton polyhedron. If $J \subseteq \mathcal{F}\left(\Gamma_{+}\right)$, we say that $S$ satisfies the $\left(C_{J}\right)$ condition when

$$
\left\{x \in \mathbb{R}^{n}: p_{J}\left(g_{1}\right)(x)=\ldots=p_{J}\left(g_{s}\right)(x)=0\right\} \subseteq\left\{x \in \mathbb{R}^{n}: x_{1} \ldots x_{n}=0\right\} .
$$

We say that $S$ is a system adapted to $\Gamma_{+}$if $S$ satisfies the $\left(C_{J}\right)$ condition for each $J \subseteq \mathcal{F}\left(\Gamma_{+}\right)$such that $\bigcap_{v \in J} \Delta\left(v, \Gamma_{+}\right)$is a compact face of $\Gamma_{+}$.

The analogous definition can be given for systems $S=\left\{g_{1}, \ldots, g_{s}\right\}$ contained in the ring $\mathcal{O}_{n}$ of complex-analytic map germs $\left(\mathbb{C}^{n}, 0\right) \rightarrow \mathbb{C}$; in this case, we replace $\mathbb{R}^{n}$ by $\mathbb{C}^{n}$ in (1). It is clear that, if $S=\left\{g_{1}, \ldots, g_{s}\right\} \subseteq \mathcal{A}_{n}$ contains some monomial $x^{k}=x_{1}^{k_{1}} \ldots x_{n}^{k_{n}}$, then $S$ is adapted to any Newton polyhedron $\Gamma_{+} \subseteq \mathbb{R}^{n}$.

Until the end of this section, we shall denote by $\Gamma_{+}$a Newton polyhedron in $\mathbb{R}^{n}$ intersecting each coordinate axis. If $L \subseteq\{1, \ldots, n\}$, we define $\mathbb{R}_{L}^{n}=$ $\left\{x \in \mathbb{R}^{n}: x_{j}=0, \forall j \in L\right\}$ and $\left(\Gamma_{+}\right)_{L}=\Gamma_{+} \cap \mathbb{R}_{L}^{n}$. We observe that $\left(\Gamma_{+}\right)_{L}$ is again a Newton polyhedron. If $g=\sum_{k} a_{k} x^{k} \in \mathcal{A}_{n}$, we denote by $g_{L}$ the series obtained as the sum of those $a_{k} x^{k}$ such that $k \in \operatorname{supp}(g) \cap \mathbb{R}_{L}^{n}$. If $\operatorname{supp}(g) \cap \mathbb{R}_{L}^{n}=\emptyset$, we set $g_{L}=0$. If $S=\left\{g_{1}, \ldots, g_{s}\right\} \subseteq \mathcal{A}_{n}$, then we write $S_{L}=\left\{\left(g_{1}\right)_{L}, \ldots,\left(g_{s}\right)_{L}\right\} \subseteq \mathcal{A}_{n-|L|}$, where $|L|$ is the cardinality of $L$. We also denote by $\left\langle g_{1}, \ldots, g_{s}\right\rangle$ the ideal of $\mathcal{A}_{n}$ generated by $g_{1}, \ldots, g_{s}$.

Definition $2.4([2])$. We say that $S$ is a system strongly adapted to $\Gamma_{+}$ if $S_{L}$ is adapted to $\left(\Gamma_{+}\right)_{L}$ for each $L \subseteq\{1, \ldots, n\}$ such that $L \neq\{1, \ldots, n\}$ (including $L=\emptyset)$. An analytic map germ $g=\left(g_{1}, \ldots, g_{s}\right):\left(\mathbb{R}^{n}, 0\right) \rightarrow\left(\mathbb{R}^{s}, 0\right)$ is said to be strongly adapted to $\Gamma_{+}$if the system $\left\{g_{1}, \ldots, g_{s}\right\}$ is strongly adapted to $\Gamma_{+}$.

Obviously, every system strongly adapted to $\Gamma_{+}$is adapted to $\Gamma_{+}$. We observe that if $S$ is adapted to $\Gamma_{+}$and $\Gamma_{+}\left(g_{i}\right)$ intersects each coordinate axis for all $i=1, \ldots, s$, then $S$ is strongly adapted to $\Gamma_{+}$.

EXAMPLE 2.5. Let $f:\left(\mathbb{R}^{2}, 0\right) \rightarrow\left(\mathbb{R}^{2}, 0\right)$ be the map defined by $f(x, y)=$ $\left(f_{1}(x, y), f_{2}(x, y)\right)=\left(x^{12}+x^{5} y+x^{3} y^{2}+x^{2} y^{3}+x y^{5}+y^{12}, x y^{9}+x^{9} y\right)$. Then $f$ is adapted to the Newton polyhedron $\Gamma_{+}\left(f_{1}\right)$. It is also strongly adapted to $\Gamma_{+}\left(f_{1}\right)$, since the support of $f_{1}$ intersects each coordinate axis.

EXAMPLE 2.6. Let $S=\left\{x^{7}+x^{2} y-x y^{2}, y^{7}+x^{2} y-x y^{2}\right\} \subseteq \mathcal{A}_{2}$. This system is not adapted to $\Gamma_{+}(S)$. Let $S^{\prime}=S \cup\left\{z^{7}\right\} \subseteq \mathcal{A}_{3}$, where we denote by $x, y, z$ the coordinates in $\mathbb{R}^{3}$. Then $S^{\prime}$ is adapted to $\Gamma_{+}\left(S^{\prime}\right)$, since $S^{\prime}$ contains a monomial; but $S^{\prime}$ is not strongly adapted, because $S=S_{L}^{\prime}$, where $L=\{3\}$. 
The proof of the following proposition is given in the next section, after introducing the notion of integral closure.

Proposition 2.7. Let $f=g+G:\left(\mathbb{R}^{n}, 0\right) \rightarrow\left(\mathbb{R}^{n}, 0\right)$ be a semi-weightedhomogeneous map germ, where $g$ is the weighted homogeneous part of $f$. Let $\mathbf{w}=\left(w_{1}, \ldots, w_{n}\right)$ be the vector of weights of $g$. Then $f$ is strongly adapted to the Newton polyhedron determined by $\left\{w_{i}^{-1} e_{i}: i=1, \ldots, n\right\}$.

We now state the main result of this paper; its proof is given in the next section.

Theorem 2.8. Let $g:\left(\mathbb{R}^{n}, 0\right) \rightarrow\left(\mathbb{R}^{n}, 0\right)$ be an analytic map germ such that $g^{-1}(0)=\{0\}$. Suppose that $g$ is strongly adapted to $\Gamma_{+}$. Let $G$ : $\left(\mathbb{R}^{n}, 0\right) \rightarrow\left(\mathbb{R}^{n}, 0\right)$ be another analytic map germ such that $\ell\left(v, G_{i}\right)>\ell\left(v, g_{i}\right)$ for all $i=1, \ldots, n$ and all $v \in \mathcal{F}_{\mathrm{c}}\left(\Gamma_{+}\right)$. Then $(g+G)^{-1}(0)=\{0\}$ and $\operatorname{ind}_{0}(g)=\operatorname{ind}_{0}(g+G)$.

As we shall see in the last section of the paper, the above result has special applications to the problem of determining which monomials can be added to each component of an analytic map $g:\left(\mathbb{R}^{2}, 0\right) \rightarrow\left(\mathbb{R}^{2}, 0\right)$ with $g^{-1}(0)=\{0\}$ without changing the index.

3. Adapted systems and the integral closure of ideals. Now, we give the definition of the integral closure of an ideal in $\mathcal{A}_{n}$. This notion plays a fundamental role in the proof of Theorem 2.8.

Definition 3.1 ([9, p. 318]). Let $I=\left\langle g_{1}, \ldots, g_{s}\right\rangle \subseteq \mathcal{A}_{n}$ be an ideal and let $h \in \mathcal{A}_{n}$. We say that $h$ is integral over $I$ if there exists an open neighbourhood $U$ of 0 in $\mathbb{R}^{n}$ and a constant $C>0$ such that

$$
|h(x)| \leq C \sup _{i}\left|g_{i}(x)\right| \quad \text { for all } x \in U .
$$

It is easy to see that this definition does not depend on the chosen system of generators of $I$. The set of elements of $\mathcal{A}_{n}$ which are integral over $I$ forms an ideal $\bar{I}$ of $\mathcal{A}_{n}$ containing $I$, called the integral closure of $I$. It is obvious that $I \subseteq \bar{I}$.

The above definitions can be reproduced analogously for ideals in the ring $\mathcal{O}_{n}$ of analytic function germs $f:\left(\mathbb{C}^{n}, 0\right) \rightarrow \mathbb{C}$ (in this case, the inequality (2) must be satisfied in an open neighbourhood of 0 in $\left.\mathbb{C}^{n}\right)$. As can be seen in [15] and [20, p. 289], if $I$ is an ideal of $\mathcal{O}_{n}$ and $h \in \mathcal{O}_{n}$, then $h \in \bar{I}$ if and only if $h$ satisfies a relation of the form $h^{m}+a_{1} h^{m-1}+\ldots+a_{m-1} h+a_{m}=0$ for some $m \geq 1$ and some $a_{i} \in I^{i}, i=1, \ldots, m$.

Theorem 3.2 ([16, p. 136]). Let $f, g \in \mathcal{A}_{n}$ be such that $g^{-1}(0) \subseteq f^{-1}(0)$. Then there exists an open neighbourhood $U$ of 0 in $\mathbb{R}^{n}$ and positive constants 
$C$ and $\alpha$ such that

$$
|f(x)|^{\alpha} \leq C|g(x)| \quad \text { for all } x \in U .
$$

The above result is also found in [4] and in [19], where it is proved that the minimum of $\alpha>0$ satisfying (3), for some constant $C>0$ and all $x$ in some neighbourhood of 0 , is a rational number. This number is called the Eojasiewicz exponent of $f$ with respect to $g$.

If $f:\left(\mathbb{R}^{n}, 0\right) \rightarrow\left(\mathbb{R}^{n}, 0\right)$ is an analytic map germ, then we shall write $I_{f}$ for the ideal of $\mathcal{A}_{n}$ generated by the component functions of $f$. We also denote by $|f|$ the function given by $|f(x)|^{2}=f_{1}(x)^{2}+\ldots+f_{p}(x)^{2}$.

DeFinition 3.3. If $f:\left(\mathbb{R}^{n}, 0\right) \rightarrow\left(\mathbb{R}^{n}, 0\right)$ is an analytic map germ such that $f^{-1}(0)=\{0\}$, then we can consider, by Theorem 3.2, the minimum of those $\alpha>0$ such that

$$
|x|^{\alpha} \leq C|f(x)|
$$

for all $x$ in some open neighbourhood of the origin in $\mathbb{R}^{n}$. This number is the Eojasiewicz exponent of $f$ and we denote it by $\alpha_{0}(f)$.

Corollary 3.4. Let $f:\left(\mathbb{R}^{n}, 0\right) \rightarrow\left(\mathbb{R}^{n}, 0\right)$ be an analytic map germ. Then $f^{-1}(0)=\{0\}$ if and only if there exists some $\beta \geq 1$ such that $m_{n}^{\beta} \subseteq \bar{I}_{f}$.

Proof. Suppose that $f^{-1}(0)=\{0\}$. Then we can apply Theorem 3.2 to $f$ and the function $\varrho(x)=x_{1}^{2}+\ldots+x_{n}^{2}$. Hence, there exists some $\alpha>0$ such that

$$
|\varrho(x)|^{\alpha} \leq C|f(x)|
$$

for some positive constant $C$ and all $x$ in some open neighbourhood of 0 in $\mathbb{R}^{n}$. But, by the definition of integral closure, this implies that $m_{n}^{\beta} \subseteq \bar{I}_{f}$ for all integers $\beta \geq[\alpha]+1$, where $[\alpha]$ is the integer part of $\alpha$.

The converse follows from a direct application of the definition of integral closure.

If $g:\left(\mathbb{R}^{r}, 0\right) \rightarrow\left(\mathbb{R}^{n}, 0\right)$ is an analytic map germ, we denote by $g^{*}$ the ring morphism $\mathcal{A}_{n} \rightarrow \mathcal{A}_{r}$ given by $g^{*}(f)=f \circ g$ for all $f \in \mathcal{A}_{n}$.

Proof of Proposition 2.7. We have $p_{\mathbf{w}}(g+G)=g$, and $p_{e_{i}}(g+G)$ is the sum of those $k \in \operatorname{supp}(g+G)$ such that $k_{i}=0$ for $i=1, \ldots, n$. Let $J \subseteq \mathcal{F}\left(\Gamma_{+}\right)=\left\{\mathbf{w}, e_{1}, \ldots, e_{n}\right\}$ be such that $\bigcap_{v \in J} \Delta\left(v, \Gamma_{+}\right)$is a compact face of $\Gamma_{+}$. Then $\mathbf{w} \in J$ and $p_{J}\left(g_{i}+G_{i}\right)=p_{J}\left(g_{i}\right)$ for all $i=1, \ldots, n$. Thus, we have to prove that $\left\{x \in \mathbb{R}^{n}:\left(g_{1}\right)_{L}(x)=\ldots=\left(g_{n}\right)_{L}(x)=0\right\} \subseteq\left\{x \in \mathbb{R}^{n}\right.$ : $\left.x_{1} \ldots x_{n}=0\right\}$ for all $L \subseteq\{1, \ldots, n\}$ with $|L| \neq n$.

Given such an $L$, if $\{1, \ldots, n\} \backslash L=\left\{j_{1}, \ldots, j_{r}\right\}, r=n-|L|$, we consider the map $\varphi_{L}:\left(\mathbb{R}^{r}, 0\right) \rightarrow\left(\mathbb{R}^{n}, 0\right)$ such that $\varphi_{L}\left(x_{j_{1}}, \ldots, x_{j_{r}}\right)=\left(y_{1}, \ldots, y_{n}\right)$, where

$$
y_{j}= \begin{cases}x_{j_{l}} & \text { if } j=j_{l} \in\left\{j_{1}, \ldots, j_{r}\right\} \\ 0 & \text { otherwise }\end{cases}
$$


We observe that $f_{L}=f \circ \varphi_{L}=\varphi_{L}^{*}(f)$ for all $f \in \mathcal{A}_{n}$. Since $g^{-1}(0)=\{0\}$, there exists some $\beta \geq 1$ such that $m_{n}^{\beta} \subseteq \bar{I}_{g}$, by Corollary 3.4. Then, for any $L \subseteq\{1, \ldots, n\}$, we have

$$
m_{r}^{\beta}=\varphi_{L}^{*}\left(m_{n}^{\beta}\right) \subseteq \varphi_{L}^{*}\left(\bar{I}_{g}\right) \subseteq \overline{\varphi_{L}^{*}\left(I_{g}\right)}=\overline{\left\langle\left(g_{1}\right)_{L}, \ldots,\left(g_{n}\right)_{L}\right\rangle} .
$$

From Corollary 3.4, it follows that the zero set germ of $\left\langle\left(g_{1}\right)_{L}, \ldots,\left(g_{n}\right)_{L}\right\rangle$, as an ideal of $\mathcal{A}_{r}$, is $\{0\}$.

The following result, which is a direct consequence of the main result of [2], will help us to prove Theorem 2.8.

Theorem $3.5([2])$. Let $S=\left\{g_{1}, \ldots, g_{s}\right\} \subseteq \mathcal{A}_{n}$ be a system adapted to a Newton polyhedron $\Gamma_{+} \subseteq \mathbb{R}^{n}$. Let $I \subseteq \mathcal{A}_{n}$ be the ideal generated by $S$ and let $k \in \mathbb{Z}_{+}^{n}$ be such that $\langle k, v\rangle \geq \max \left\{\ell\left(v, g_{1}\right), \ldots, \ell\left(v, g_{s}\right)\right\}$ for all $v \in \mathcal{F}\left(\Gamma_{+}\right)$. Then $x^{k} \in \bar{I}$.

REMARK 3.6. In the conditions of the above theorem, if we assume that the support of each germ $g_{i}$ intersects each coordinate axis, then for each $i \in\{1, \ldots, n\}$, there exists some $k_{i} \geq 0$ such that $x_{i}^{k_{i}} \in \bar{I}$.

For $z \in \mathbb{R}^{n}$, we denote by $\mathcal{A}_{n, z}$ the ring of analytic function germs $g$ : $\left(\mathbb{R}^{n}, z\right) \rightarrow \mathbb{R}$. Suppose that $g: U \rightarrow \mathbb{R}$ is a map defined on an open set $U \subseteq \mathbb{R}^{n}$. Then we write $\gamma_{z}(g)$ for the germ of $g$ at any $z \in U$. Let $\varphi_{z}$ : $\left(\mathbb{R}^{n}, 0\right) \rightarrow\left(\mathbb{R}^{n}, z\right)$ be given by $\varphi_{z}(x)=x+z$. If $g \in \mathcal{A}_{n, z}$, we define the support of $g$ as $\operatorname{supp}(g)=\operatorname{supp}\left(g \circ \varphi_{z}\right)$. Given any $v \in \mathbb{R}_{+}^{n} \backslash\{0\}$, we also define the number $\ell(v, g)$ as $\ell\left(v, g \circ \varphi_{z}\right)$. If $\Gamma_{+} \subseteq \mathbb{R}^{n}$ is a Newton polyhedron and $S=\left\{g_{1}, \ldots, g_{s}\right\} \subseteq \mathcal{A}_{n, z}$, we say that $S$ is adapted to $\Gamma_{+}$if the system $S^{\prime}=\left\{g_{1} \circ \varphi_{z}, \ldots, g_{s} \circ \varphi_{z}\right\}$ is adapted to $\Gamma_{+}$. The following is an immediate consequence of Theorem 3.5.

Corollary 3.7. Let $z=\left(z_{1}, \ldots, z_{n}\right) \in \mathbb{R}^{n}$ and let $S=\left\{g_{1}, \ldots, g_{s}\right\} \subseteq$ $\mathcal{A}_{n, z}$ be a system adapted to a Newton polyhedron $\Gamma_{+} \subseteq \mathbb{R}^{n}$. Let $I \subseteq \mathcal{A}_{n, z}$ be the ideal generated by $S$ and let $k=\left(k_{1}, \ldots, k_{n}\right) \in \mathbb{Z}_{+}^{n}$ be such that $\langle k, v\rangle \geq \max \left\{\ell\left(v, g_{1}\right), \ldots, \ell\left(v, g_{s}\right)\right\}$ for all $v \in \mathcal{F}\left(\Gamma_{+}\right)$. Then

$$
\left(x_{1}-z_{1}\right)^{k_{1}} \ldots\left(x_{n}-z_{n}\right)^{k_{n}} \in \bar{I} .
$$

The following result is a version for real-analytic functions of the integral Nakayama lemma (see [20, p. 324] for an algebraic proof in the context of ideals in $\left.\mathcal{O}_{n}\right)$.

Lemma 3.8. Let $I, J \subseteq \mathcal{A}_{n}$ be a pair of ideals. If $I \subseteq \overline{J+m_{n} I}$, then $I \subseteq \bar{J}$.

Proof. Let $g_{1}, \ldots, g_{s}$ be a generating system for $I$ and let $h_{1}, \ldots, h_{r}$ be a generating system for $J$. The inclusion $I \subseteq \overline{J+m_{n} I}$ means that there exist 
an open neighbourhood of 0 in $\mathbb{R}^{n}$ and a constant $C>0$ such that

$$
\sup _{i}\left|g_{i}(x)\right| \leq C \sup _{j, k, l}\left\{\left|h_{j}(x)\right|,\left|x_{k} g_{l}(x)\right|\right\}
$$

for all $x \in U$. Suppose that $\bar{I} \nsubseteq \bar{J}$. Then, for any constant $B>0$, there exists a sequence $\left\{x_{m}^{B}\right\}_{m \geq 1}$ in $\mathbb{R}^{n}$ tending to 0 such that

$$
\sup _{i}\left|g_{i}\left(x_{m}^{B}\right)\right|>B \sup _{j}\left|h_{j}\left(x_{m}^{B}\right)\right| \quad \text { for all } m \geq 1 .
$$

Then, taking $B=2 C$ and $m$ large enough, we have

$$
\begin{aligned}
\sup _{i}\left|g_{i}\left(x_{m}^{2 C}\right)\right| & =\frac{C}{2 C} \sup _{i}\left|g_{i}\left(x_{m}^{2 C}\right)\right|+\frac{C}{2 C} \sup _{i}\left|g_{i}\left(x_{m}^{2 C}\right)\right| \\
& >C \sup _{j}\left|h_{j}\left(x_{m}^{2 C}\right)\right|+C\left|x_{m}^{2 C}\right| \sup _{i}\left|g_{i}\left(x_{m}^{2 C}\right)\right| \\
& \geq C \sup _{j, k, l}\left\{\left|h_{j}\left(x_{m}^{2 C}\right)\right|,\left|\left(x_{m}^{2 C}\right)_{k}\right| \cdot\left|g_{l}\left(x_{m}^{2 C}\right)\right|\right\},
\end{aligned}
$$

which contradicts (4). Therefore, $I \subseteq \bar{J}$.

If $g=\sum_{k} a_{k} x^{k}$ is the Taylor expansion of a germ $g \in \mathcal{A}_{n}$ and $r \geq 1$, we denote by $j^{r} g$ the $r$-jet of $g$, that is, the sum of those $a_{k} x^{k}$ such $|k|=$ $k_{1}+\ldots+k_{n} \leq r$. If $f:\left(\mathbb{R}^{n}, 0\right) \rightarrow\left(\mathbb{R}^{p}, 0\right)$ is an analytic map, we denote by $j^{r} f$ the map $\left(j^{r} f_{1}, \ldots, j^{r} f_{p}\right)$.

The next lemma was proved in [7, p. 31] for finite map germs, that is, for analytic map germs $f:\left(\mathbb{R}^{n}, 0\right) \rightarrow\left(\mathbb{R}^{n}, 0\right)$ such that $\operatorname{dim}_{\mathbb{R}} \mathcal{A}_{n} / I_{f}<\infty$.

Lemma 3.9. Let $f:\left(\mathbb{R}^{n}, 0\right) \rightarrow\left(\mathbb{R}^{n}, 0\right)$ be an analytic map germ with $f^{-1}(0)=\{0\}$. Then there exists some $r \geq 1$ such that if $g:\left(\mathbb{R}^{n}, 0\right) \rightarrow$ $\left(\mathbb{R}^{n}, 0\right)$ is another analytic map germ such that $j^{r} f=j^{r}$ g, then $g^{-1}(0)=\{0\}$ and $\operatorname{ind}_{0}(f)=\operatorname{ind}_{0}(g)$.

Proof. Since $f^{-1}(0)=\{0\}$, there is an integer $r \geq 0$ such that $m_{n}^{r} \subseteq \bar{I}_{f}$, by Theorem 3.2. Then we obtain

$$
I_{f} \subseteq I_{j^{r} f}+m_{n}^{r+1} \subseteq I_{j^{r} f}+m_{n} m_{n}^{r} \subseteq I_{j^{r} f}+m_{n} \bar{I}_{f} \subseteq \overline{I_{j^{r} f}+m_{n} \bar{I}_{f}} .
$$

Thus, from Lemma 3.8, we obtain the inclusion $I_{f} \subseteq \bar{I}_{j^{r} f}$. Consider now the homotopy $g_{t}=j^{r} f+t \varrho$, where $\varrho=\left(\varrho_{1}, \ldots, \varrho_{n}\right)$ and $\varrho_{i} \in m_{n}^{r+1} \subseteq m_{n} \bar{I}_{f}$ for all $i=1, \ldots, n$ and $t \in[0,1]$. We deduce that

$$
\begin{aligned}
\left|j^{r} f(x)+t \varrho(x)\right| & \geq\left|j^{r} f(x)\right|-t|\varrho(x)| \geq C_{1}|f(x)|-t C_{2}|x| \cdot|f(x)| \\
& =|f(x)|\left(C_{1}-t C_{2}|x|\right)
\end{aligned}
$$

for some constants $C_{1}, C_{2}>0$, for all $x$ in an open neighbourhood $U$ of 0 in $\mathbb{R}^{n}$ and all $t \in[0,1]$. By the above inequalities and the relation $f^{-1}(0)=\{0\}$, we can take $U$ small enough that

$$
\left\{(x, t) \in U \times[0,1]: j^{r} f(x)+t \varrho(x)=0\right\}=\{0\} \times[0,1] .
$$


But this implies that $\left(g_{t}\right)^{-1}(0)=\{0\}$ and $\operatorname{ind}_{0}(f)=\operatorname{ind}_{0}\left(g_{t}\right)$ for all $t \in[0,1]$ (see [18]).

REMARK 3.10. By the proof of the above lemma, if $f:\left(\mathbb{R}^{n}, 0\right) \rightarrow\left(\mathbb{R}^{n}, 0\right)$ is an analytic map germ such that $f^{-1}(0)=\{0\}$, then $\operatorname{ind}_{0}(f)=\operatorname{ind}_{0}\left(j^{r} f\right)$ for all $r \geq \alpha_{0}(f)$.

Lemma 3.11. Let $F:\left(\mathbb{R}^{n} \times[0,1],\{0\} \times[0,1]\right) \rightarrow\left(\mathbb{R}^{n}, 0\right)$ be an analytic map germ. If $F=\left(F_{1}, \ldots, F_{n}\right)$ and $t \in[0,1]$, let $J_{t}$ be the ideal of $\mathcal{A}_{n+1,(0, t)}$ generated by the germs $\gamma_{(0, t)}\left(F_{1}\right), \ldots, \gamma_{(0, t)}\left(F_{n}\right)$, and let $f_{t}$ : $\left(\mathbb{R}^{n}, 0\right) \rightarrow\left(\mathbb{R}^{n}, 0\right)$ be given by $f_{t}(x)=F(t, x)$. Suppose that there exists some $N>0$ such that $x_{i}^{N} \in \bar{J}_{t}$ for all $t \in[0,1]$ and $i \in\{1, \ldots, n\}$. Then 0 is isolated in $f_{t}^{-1}(0)$ and $\operatorname{ind}_{0}\left(f_{0}\right)=\operatorname{ind}_{0}\left(f_{t}\right)$ for all $t \in[0,1]$.

Proof. By hypothesis, given any $t_{0} \in[0,1]$, the function $\varrho(x)=x_{1}^{2 N}+$ $\ldots+x_{n}^{2 N}$ belongs to $\bar{J}_{t_{0}}$. But this means that there exist open neighbourhoods $U_{t_{0}} \subseteq \mathbb{R}^{n}$ and $V_{t_{0}} \subseteq \mathbb{R}$ of 0 and $t_{0}$ respectively such that

$$
|\varrho(x)| \leq C_{t_{0}} \sup _{i}\left|F_{i}(x, t)\right|
$$

for all $(x, t) \in U_{t_{0}} \times V_{t_{0}}$ and some constant $C_{t_{0}}>0$.

By the compactness of $[0,1]$, there exist $t_{1}, \ldots, t_{s} \in[0,1]$ such that $[0,1] \subseteq V_{t_{1}} \cup \ldots \cup V_{t_{s}}$. Let $C=\max \left\{C_{t_{1}}, \ldots, C_{t_{s}}\right\}$ and $U=U_{t_{1}} \cap \ldots \cap U_{t_{s}}$. Then

$$
|\varrho(x)| \leq C \sup _{i}\left|F_{i}(x, t)\right|
$$

for all $(x, t) \in U \times[0,1]$. In particular,

$$
\left\{(x, t) \in U \times[0,1]: F_{i}(x, t)=0, \forall i=1, \ldots, n\right\}=\{0\} \times[0,1],
$$

which implies the desired result.

Proof of Theorem 2.8. Consider the function $\theta(x)=\sum_{i=1}^{n} x_{i}^{M}$, where $M$ is a positive number, and the map $g^{*}=\left(g_{1}+\theta, \ldots, g_{n}+\theta\right)$. If $M$ is large enough, we can $\operatorname{suppose}$ that $\operatorname{supp}\left(g_{i}+\theta\right)=\operatorname{supp}\left(g_{i}\right) \cup \operatorname{supp}(\theta)$ for $i=1, \ldots, n$, and that $\operatorname{ind}_{0}(g)=\operatorname{ind}_{0}\left(g^{*}\right)$, by Lemma 3.9. Let us see that $g^{*}$ is adapted to $\Gamma_{+}$. If $v=\left(v_{1}, \ldots, v_{n}\right) \in \mathcal{F}_{\mathrm{c}}\left(\Gamma_{+}\right)$and $i \in\{1, \ldots, n\}$, then

$$
\begin{aligned}
\ell\left(v, g_{i}+\theta\right)=\ell\left(v, g_{i}\right) & \Leftrightarrow \ell\left(v, g_{i}\right) \leq \min \{\langle k, v\rangle: k \in \operatorname{supp}(\theta)\} \\
& \Leftrightarrow \ell\left(v, g_{i}\right) \leq \min \left\{M v_{j}: j=1, \ldots, n\right\} \\
& \Leftrightarrow \frac{\ell\left(v, g_{i}\right)}{\min \left\{v_{j}: j=1, \ldots, n\right\}} \leq M .
\end{aligned}
$$

By the above observation, if we take $M$ large enough, we conclude that $\ell\left(v, g_{i}+\theta\right)=\ell\left(v, g_{i}\right)$ and $\Delta\left(v, g_{i}+\theta\right)=\Delta\left(v, g_{i}\right)$ for all $v \in \mathcal{F}_{\mathrm{c}}\left(\Gamma_{+}\right)$, and $\ell\left(e_{j}, g_{i}+\theta\right)=0$ for all $i, j \in\{1, \ldots, n\}$. If $J \subseteq \mathcal{F}\left(\Gamma_{+}\right)$is such that $\bigcap_{v \in J} \Delta\left(v, \Gamma_{+}\right)$is a compact face of $\Gamma_{+}$then, defining $L_{J}=\left\{i: e_{i} \in J\right\}$, we 
obtain

$$
p_{J}\left(g_{i}+\theta\right)=p_{J}\left(\left(g_{i}+\theta\right)_{L_{J}}\right)=p_{J}\left(\left(g_{i}\right)_{L_{J}}\right) .
$$

Combining this with the hypothesis that $S$ is strongly adapted to $\Gamma_{+}$, we find that the system $\left\{g_{1}+\theta, \ldots, g_{n}+\theta\right\}$ is adapted to $\Gamma_{+}$.

Let $F:\left(\mathbb{R}^{n} \times[0,1],\{0\} \times[0,1]\right) \rightarrow\left(\mathbb{R}^{n}, 0\right)$ be the map germ defined by $F(x, t)=g^{*}(x)+t G(x)$. For each $t \in[0,1]$, we denote by $J_{t}$ the ideal in $\mathcal{A}_{n+1,(0, t)}$ generated by $\gamma_{(0, t)}\left(F_{1}\right), \ldots, \gamma_{(0, t)}\left(F_{n}\right)$, where $F_{1}, \ldots, F_{n}$ are the coordinate functions of $F$. We will prove that there exists some $N>0$ such that $x_{i}^{N} \in \bar{J}_{t}$ for all $t \in[0,1]$ and $i \in\{1, \ldots, n\}$, in order to apply Lemma 3.11.

Consider the Newton polyhedron $\Gamma_{+}^{\prime}=\Gamma_{+} \times \mathbb{R}_{+}$(we recall that $\Gamma_{+}$is a Newton polyhedron intersecting all the coordinate axes). We claim that the system $S_{t}=\left\{\gamma_{(0, t)}\left(F_{1}\right), \ldots, \gamma_{(0, t)}\left(F_{n}\right)\right\}$ is adapted to $\Gamma_{+}^{\prime}$ for all $t \in[0,1]$. Let $p_{a}: \mathbb{R}^{n} \rightarrow \mathbb{R}^{n+1}$ be given by $p_{a}\left(k_{1}, \ldots, k_{n}\right)=\left(k_{1}, \ldots, k_{n}, a\right)$, where $a \in \mathbb{R}$. For any $i \in\{1, \ldots, n\}$, we observe that

$$
\begin{aligned}
& \operatorname{supp}\left(\gamma_{(0, t)}\left(F_{i}\right)\right) \\
& \quad= \begin{cases}p_{0}\left(\operatorname{supp}\left(g_{i}+\theta\right)\right) \cup p_{0}\left(\operatorname{supp}\left(G_{i}\right)\right) \cup p_{1}\left(\operatorname{supp}\left(G_{i}\right)\right) & \text { if } t \neq 0, \\
p_{0}\left(\operatorname{supp}\left(g_{i}+\theta\right)\right) \cup p_{1}\left(\operatorname{supp}\left(G_{i}\right)\right) & \text { if } t=0 .\end{cases}
\end{aligned}
$$

We also have $\mathcal{F}_{\mathrm{c}}\left(\Gamma_{+}^{\prime}\right)=\emptyset$ and

$$
\mathcal{F}\left(\Gamma_{+}^{\prime}\right)=\left\{p_{0}(v): v \in \mathcal{F}\left(\Gamma_{+}\right)\right\} \cup\{(0, \ldots, 0,1)\}=\left\{p_{0}(v): v \in \mathcal{F}_{\mathrm{c}}\left(\Gamma_{+}\right)\right\} \cup \mathcal{B},
$$

where $\mathcal{B}$ denotes the canonical basis in $\mathbb{R}^{n+1}$.

Fix some $t \in[0,1]$, a vector $v \in \mathcal{F}_{\mathrm{c}}\left(\Gamma_{+}\right)$and an index $i \in\{1, \ldots, n\}$. Then the condition $\ell\left(v, G_{i}\right)>\ell\left(v, g_{i}\right)$, together with (6), implies that

$$
\ell\left(p_{0}(v), \gamma_{(0, t)}\left(F_{i}\right)\right)=\ell\left(p_{0}(v), \gamma_{(0, t)}\left(g_{i}+\theta\right)\right)=\ell\left(v, g_{i}+\theta\right),
$$

where, in the first equality, we are considering $g_{i}+\theta$ as a germ in $\mathcal{A}_{(0, t)}$ in the obvious way.

Moreover, it is clear that

$$
\ell\left(u, \gamma_{(0, t)}\left(F_{i}\right)\right)=0 \quad \text { for all } u \in \mathcal{B} .
$$

Hence, the principal parts of $\gamma_{(0, t)}\left(F_{i}\right)$ and $\gamma_{(0, t)}\left(g_{i}+\theta\right)$ with respect to any vector of $\mathcal{F}\left(\Gamma_{+}^{\prime}\right)$ are equal. Thus, the principal part of $\gamma_{(0, t)}\left(F_{i}\right)$ with respect to a subset $J \subseteq \mathcal{F}\left(\Gamma_{+}^{\prime}\right)$ (see Definition 2.2) is equal to the principal part of $\gamma_{(0, t)}\left(g_{i}+\theta\right)$ with respect to $J$, for all $t \in[0,1]$. This implies that the system $S_{t}$ is adapted to $\Gamma_{+}^{\prime}$, since $\left\{g_{1}+\theta, \ldots, g_{n}+\theta\right\}$ is adapted to $\Gamma_{+}$.

Therefore, from the identities (7) and (8) and Corollary 3.7, there is a sufficiently large positive integer $N$ such that $x_{i}^{N} \in \bar{J}_{t} \subseteq \mathcal{A}_{n+1,(0, t)}$ for all $i=1, \ldots, n$ (see Remark 3.6). Hence, the result follows from Lemma 3.11.

ExAmple $3.12([6])$. Let $g:\left(\mathbb{R}^{2}, 0\right) \rightarrow\left(\mathbb{R}^{2}, 0\right)$ be given by $g(x, y)=$ $\left(y^{2}, y-x^{4}\right)$, and $f(x, y)=g(x, y)+\left(-x^{3} y, 0\right)$. The map $g$ is weighted homo- 
geneous of weights 1,4 and degrees 8,4 . Moreover $\operatorname{ind}_{0}(g)=0$ and $\operatorname{ind}_{0}(f)=$ -1 . Let us see explicitly that the deformation $F(x, t)=g(x)+t\left(-x^{3} y, 0\right)$ does not satisfy the conditions of Lemma 3.11.

Let $J_{0}$ be the ideal of $\mathcal{A}_{3}$ generated by $S=\left\{y^{2}-t x^{3} y, y-x^{4}\right\}$. Suppose that there exists some $N>0$ such that $x^{N}, y^{N} \in \bar{J}_{0}$. In particular, there exists some open neighbourhood $U$ of 0 in $\mathbb{R}^{3}$ and a constant $C>0$ such that

$$
\left|x^{N}\right| \leq C \sup \left\{\left|y^{2}-t x^{3} y\right|,\left|y-x^{4}\right|\right\}
$$

for all $(x, y, t) \in U$. But the right hand side of (9) vanishes on the curve $\left\{(x, y, t): x=t, y=x^{4}\right\}$, which passes through the origin. Thus, we have a contradiction.

EXAMPLE 3.13. Let $g:\left(\mathbb{R}^{2}, 0\right) \rightarrow\left(\mathbb{R}^{2}, 0\right)$ be given by $g(x, y)=\left(x^{a}+y^{a}+\right.$ $\left.x^{a-4} y^{2}+x^{2} y^{a-4}, x y^{a-2}+x^{a-2} y\right)$, where $a \geq 7$. Then $g$ is strongly adapted to $\Gamma_{+}=\Gamma_{+}(g)$. Here is a picture of $\Gamma_{+}(g)$ :

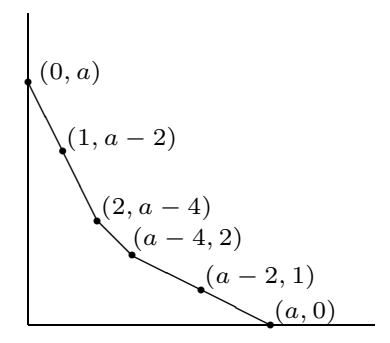

Let $\Gamma_{+}^{\prime}$ be the Newton polyhedron determined by the points $(a, 0),(a-2,1)$, $(1, a-2)$ and $(0, a)$. By Theorem 2.8 any analytic map germ $G=\left(G_{1}, G_{2}\right)$ : $\left(\mathbb{R}^{2}, 0\right) \rightarrow\left(\mathbb{R}^{2}, 0\right)$ such that $\operatorname{supp}\left(G_{1}\right) \subseteq \Gamma_{+} \backslash \Gamma$ and $\operatorname{supp}\left(G_{2}\right) \subseteq \Gamma_{+}^{\prime} \backslash \Gamma^{\prime}$ satisfies $(g+G)^{-1}(0)=\{0\}$ and $\operatorname{ind}_{0}(g)=\operatorname{ind}_{0}(g+G)$.

EXAMPLE 3.14. Let $g:\left(\mathbb{R}^{3}, 0\right) \rightarrow\left(\mathbb{R}^{3}, 0\right)$ be given by $g(x, y, z)=\left(x^{a}+z^{c}\right.$, $\left.y^{b}-y^{2} z^{2}+z^{c}, x^{a}+y^{b}+y^{2} z^{2}\right)$, where $a>0$ and $b, c>4$. Let $g_{1}, g_{2}, g_{3}$ be the components of $g$ and let $\Gamma_{+} \subseteq \mathbb{R}^{3}$ be the Newton polyhedron determined by them. Let $\Gamma$ be the union of the compact faces of $\Gamma_{+}$. Here is what $\Gamma$ looks like:

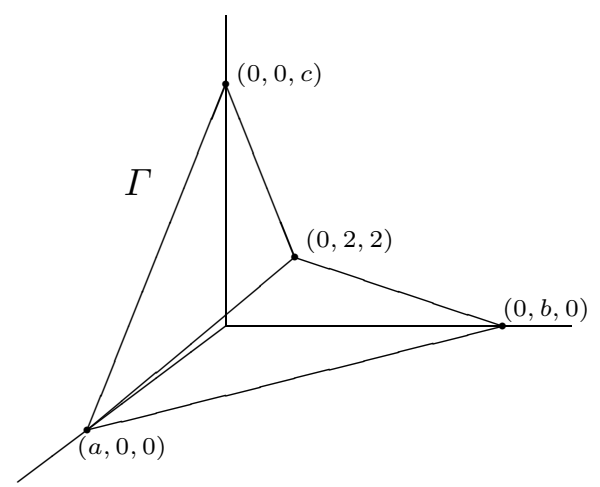


The map $g$ is strongly adapted to $\Gamma_{+}$and $\ell\left(v, g_{1}\right)=\ell\left(v, g_{2}\right)=\ell\left(v, g_{3}\right)$ for all $v \in \mathcal{F}_{\mathrm{c}}\left(\Gamma_{+}\right)$. Suppose that $g^{-1}(0)=\{0\}$ and $G=\left(G_{1}, G_{2}, G_{3}\right):\left(\mathbb{R}^{3}, 0\right) \rightarrow$ $\left(\mathbb{R}^{3}, 0\right)$ is another analytic map germ such that $\operatorname{supp}\left(G_{i}\right) \subseteq \Gamma_{+} \backslash \Gamma, i=1,2,3$. Then, by Theorem 2.8, $(g+G)^{-1}(0)=\{0\}$ and $\operatorname{ind}_{0}(g)=\operatorname{ind}_{0}(g+G)$.

4. Non-degenerate maps. In this section, we introduce the concept of strongly non-degenerate maps $\left(\mathbb{R}^{n}, 0\right) \rightarrow\left(\mathbb{R}^{n}, 0\right)$, which is motivated by the notion of non-degenerate complete intersection given by Khovanskiu (see [9, p. 315] or [14]). We obtain an analogue of Theorem 2.8 for the index of strongly non-degenerate maps, which generalizes a result of Gutiérrez-Ruas [13] about the index of germs of vector fields in $\mathbb{R}^{2}$.

Definition 4.1. Let $S=\left\{g_{1}, \ldots, g_{s}\right\} \subseteq \mathcal{A}_{n}$ and $v \in \mathbb{R}_{+}^{n} \backslash\{0\}$. We say that $S$ satisfies the $\left(K_{v}\right)$ condition if

$$
\left\{x \in \mathbb{R}^{n}: p_{v}\left(g_{1}\right)(x)=\ldots=p_{v}\left(g_{s}\right)(x)=0\right\} \subseteq\left\{x \in \mathbb{R}^{n}: x_{1} \ldots x_{n}=0\right\} .
$$

The system $S=\left\{g_{1}, \ldots, g_{s}\right\} \subseteq \mathcal{A}_{n}$ is said to be non-degenerate if $S$ satisfies the $\left(K_{v}\right)$ condition for all $v \in \mathbb{R}_{+}^{n} \backslash\{0\}$. If $g=\left(g_{1}, \ldots, g_{s}\right):\left(\mathbb{R}^{n}, 0\right) \rightarrow\left(\mathbb{R}^{s}, 0\right)$ is an analytic map germ, we say that $g$ is non-degenerate if the system $\left\{g_{1}, \ldots, g_{s}\right\}$ is non-degenerate.

We need some preliminary lemmas in order to relate non-degenerate systems and systems adapted to Newton polyhedra. If $\Gamma_{+}^{1}, \ldots, \Gamma_{+}^{s}$ are Newton polyhedra in $\mathbb{R}^{n}$, their Minkowski sum, denoted by $\Gamma_{+}^{1}+\ldots+\Gamma_{+}^{s}$, is the Newton polyhedron given by $\left\{k_{1}+\ldots+k_{s}: k_{i} \in \Gamma_{+}^{i}, i=1, \ldots, s\right\}$.

The proof of the following result can be found in [8, p. 77].

Lemma 4.2. Let $\Gamma_{+}$be the Minkowski sum of the Newton polyhedra $\Gamma_{+}^{1}, \ldots, \Gamma_{+}^{s} \subseteq \mathbb{R}^{n}$ and let $v, v^{\prime} \in \mathbb{R}_{+}^{n} \backslash\{0\}$. Then

(1) $\ell\left(v, \Gamma_{+}\right)=\ell\left(v, \Gamma_{+}^{1}\right)+\ldots+\ell\left(v, \Gamma_{+}^{s}\right)$;

(2) $\Delta\left(v, \Gamma_{+}\right)=\Delta\left(v, \Gamma_{+}^{1}\right)+\ldots+\Delta\left(v, \Gamma_{+}^{s}\right)$;

(3) $\Delta\left(v, \Gamma_{+}\right)=\Delta\left(v^{\prime}, \Gamma_{+}\right)$if and only if $\Delta\left(v, \Gamma_{+}^{i}\right)=\Delta\left(v^{\prime}, \Gamma_{+}^{i}\right)$ for all $i=1, \ldots, s$.

Lemma 4.3. Let $\Gamma_{+} \subseteq \mathbb{R}^{n}$ be a Newton polyhedron and let $J \subseteq \mathbb{R}_{+}^{n} \backslash\{0\}$ be a finite subset. Then the following assertions are equivalent:

(1) $\ell\left(\sum_{v \in J} v, \Gamma_{+}\right)=\sum_{v \in J} \ell\left(v, \Gamma_{+}\right)$;

(2) $\Delta\left(\sum_{v \in J} v, \Gamma_{+}\right)=\bigcap_{v \in J} \Delta\left(v, \Gamma_{+}\right)$;

(3) $\bigcap_{v \in J} \Delta\left(v, \Gamma_{+}\right) \neq \emptyset$.

Proof. This follows easily from the definitions.

Now, we give a technical lemma that we will use in the proof of the next proposition. This lemma says that in the definition of a system adapted to a given Newton polyhedron $\Gamma_{+}$(see Definition 2.3), the conditions $\left(C_{J}\right)$ can be attached to arbitrary faces of $\Gamma_{+}$. 
Lemma 4.4. Let $\Gamma_{+} \subseteq \mathbb{R}^{n}$ be a Newton polyhedron. Then the system $S=\left\{g_{1}, \ldots, g_{s}\right\} \subseteq \mathcal{A}_{n}$ is adapted to $\Gamma_{+}$if and only if $S$ satisfies the $\left(C_{J}\right)$ condition for any $J \subseteq \mathcal{F}\left(\Gamma_{+}\right)$such that $\bigcap_{j \in J} \Delta\left(v^{j}, \Gamma_{+}\right)$is a (not necessarily compact) face of $\Gamma_{+}$.

Proof. The "if" part is obvious. Let us see the other implication. Let $J \subseteq \mathcal{F}\left(\Gamma_{+}\right)$be such that $\bigcap_{v \in J} \Delta\left(v, \Gamma_{+}\right)$is an arbitrary face of $\Gamma_{+}$(not necessarily compact). Then the system $\left\{p_{J}\left(g_{1}\right), \ldots, p_{J}\left(g_{s}\right)\right\}$ is adapted to $\Gamma_{+}$. But, by Theorem 3.5, there exists some monomial $x^{k}$ belonging to the integral closure of the ideal in $\mathcal{A}_{n}$ generated by $\left\{p_{J}\left(g_{1}\right), \ldots, p_{J}\left(g_{s}\right)\right\}$. In particular, by Definition 3.1, the zero set of $\left\{p_{J}\left(g_{1}\right), \ldots, p_{J}\left(g_{s}\right)\right\}$ is contained in $\left\{x \in \mathbb{R}^{n}: x_{1} \ldots x_{n}=0\right\}$.

Proposition $4.5([2])$. Let $S=\left\{g_{1}, \ldots, g_{s}\right\} \subseteq \mathcal{A}_{n}$. Then $S$ is non-degenerate if and only if $S$ is adapted to the Newton polyhedron $\Gamma_{+}=\Gamma_{+}\left(g_{1}\right)+$ $\ldots+\Gamma_{+}\left(g_{s}\right)$.

Proof. Suppose that $S$ is adapted to $\Gamma_{+}$. Let $\mathcal{F}\left(\Gamma_{+}\right)=\left\{v^{1}, \ldots, v^{r}\right\}$. If $v \in \mathbb{R}_{+}^{n} \backslash\{0\}$, then $\Delta\left(v, \Gamma_{+}\right)=\bigcap_{j \in J} \Delta\left(v^{j}, \Gamma_{+}\right)$for some $J \subseteq\{1, \ldots, r\}$. Thus, there exist $k_{i} \in \Gamma_{+}\left(g_{i}\right)$ for $i=1, \ldots, s$ such that

$$
\left\langle k_{1}+\ldots+k_{s}, v^{j}\right\rangle=\ell\left(v^{j}, \Gamma_{+}\right), \quad \forall j \in J .
$$

But, by Lemma $4.2(1)$, the above relation is equivalent to $\left\langle k_{i}, v^{j}\right\rangle=\ell\left(v^{j}, g_{i}\right)$ for all $i \in\{1, \ldots, s\}, j \in J$. In particular, this means that $\bigcap_{j \in J} \Delta\left(v^{j}, g_{i}\right) \neq \emptyset$ for all $i \in\{1, \ldots, s\}$. Therefore, from Lemma 4.3, we have

$$
\bigcap_{j \in J} \Delta\left(v^{j}, g_{i}\right)=\Delta\left(\sum_{j \in J} v^{j}, g_{i}\right) \quad \text { for all } i \in\{1, \ldots, s\} .
$$

If $w=\sum_{j \in J} v^{j}$, the above equality implies that $p_{J}\left(g_{i}\right)=p_{w}\left(g_{i}\right)$ for all $i=1, \ldots, s$. Moreover, the fact that $\bigcap_{j \in J} \Delta\left(v^{j}, \Gamma_{+}\right) \neq \emptyset$ also implies that

$$
\Delta\left(v, \Gamma_{+}\right)=\bigcap_{j \in J} \Delta\left(v^{j}, \Gamma_{+}\right)=\Delta\left(\sum_{j \in J} v^{j}, \Gamma_{+}\right)=\Delta\left(w, \Gamma_{+}\right) .
$$

Then, from Lemma $4.2(3)$, we have $\Delta\left(v, \Gamma_{+}\left(g_{i}\right)\right)=\Delta\left(w, \Gamma_{+}\left(g_{i}\right)\right)$ for all $i=1, \ldots, s$. But this means that $p_{v}\left(g_{i}\right)=p_{w}\left(g_{i}\right)=p_{J}\left(g_{i}\right)$ for all $i=1, \ldots, s$. Hence, by Lemma 4.4 , we conclude that $S$ satisfies the $\left(K_{v}\right)$ condition.

Suppose that $S$ is non-degenerate. Let $J \subseteq\{1, \ldots, r\}$ be such that $\bigcap_{j \in J} \Delta\left(v^{j}, \Gamma_{+}\right)$is a face of $\Gamma_{+}$. Then, if we define $w=\sum_{j \in J} v^{j}$, by an argument similar to the previous one, we also deduce that $p_{J}\left(g_{i}\right)=p_{w}\left(g_{i}\right)$ for all $i \in\{1, \ldots, s\}$. Thus $S$ satisfies the $\left(C_{J}\right)$ condition.

The next definition is analogous to Definition 2.4.

Definition 4.6. The system $S=\left\{g_{1}, \ldots, g_{s}\right\} \subseteq \mathcal{A}_{n}$ is said to be strongly non-degenerate if, for any $L \subseteq\{1, \ldots, n\}$ with $|L| \neq n$, the system $S_{L}$ is non-degenerate as a system in $\mathcal{A}_{n-|L|}$. 
Let $S=\left\{g_{1}, \ldots, g_{s}\right\} \subseteq \mathcal{A}_{n}$. Then it is straightforward that if $\Gamma_{+}\left(g_{i}\right)$ intersects each coordinate axis for all $i=1, \ldots, s$, then $S$ is non-degenerate if and only if $S$ is strongly non-degenerate.

COROLlary 4.7. Let $g:\left(\mathbb{R}^{n}, 0\right) \rightarrow\left(\mathbb{R}^{n}, 0\right)$ be a non-degenerate analytic map germ such that $g^{-1}(0)=\{0\}$ and $\Gamma_{+}\left(g_{i}\right)$ intersects each coordinate axis for all $i=1, \ldots, s$. Suppose that $G:\left(\mathbb{R}^{n}, 0\right) \rightarrow\left(\mathbb{R}^{n}, 0\right)$ is an analytic map germ such that $\ell\left(v, G_{i}\right)>\ell\left(v, g_{i}\right)$ for all $i=1, \ldots, n$ and all $v \in \mathcal{F}_{\mathrm{c}}\left(\Gamma_{+}\right)$, where $\Gamma_{+}=\Gamma_{+}\left(g_{1}\right)+\ldots+\Gamma_{+}\left(g_{s}\right)$. Then

$$
(g+G)^{-1}(0)=\{0\} \quad \text { and } \operatorname{ind}_{0}(g)=\operatorname{ind}_{0}(g+G) .
$$

Proof. The Minkowski sum $\Gamma_{+}=\Gamma_{+}\left(g_{1}\right)+\ldots+\Gamma_{+}\left(g_{s}\right)$ is again a Newton polyhedron intersecting each coordinate axis, so $\left(\Gamma_{+}\right)_{L} \neq \emptyset$ for all $L \subseteq$ $\{1, \ldots, n\}$ with $|L| \neq n$. By Proposition $4.5, g$ is strongly non-degenerate if and only if it is strongly adapted to $\Gamma_{+}$. Now the result follows from Theorem 2.8.

COROLlaRY 4.8. Let $g:\left(\mathbb{R}^{n}, 0\right) \rightarrow\left(\mathbb{R}^{n}, 0\right)$ be a strongly non-degenerate analytic map germ such that $g^{-1}(0)=\{0\}$. Let $G:\left(\mathbb{R}^{n}, 0\right) \rightarrow\left(\mathbb{R}^{n}, 0\right)$ be an analytic map germ such that $\operatorname{supp}\left(G_{i}\right) \subseteq \Gamma_{+}\left(g_{i}\right) \backslash \Gamma\left(g_{i}\right)$ for all $i=1, \ldots, n$. Then $(g+G)^{-1}(0)=\{0\}$ and $\operatorname{ind}_{0}(g)=\operatorname{ind}_{0}(g+G)$.

Proof. Consider the map $\theta(x)=x_{1}^{M}+\ldots+x_{n}^{M}$, where $M$ is a positive integer, and set $h=\left(g_{1}+\theta, \ldots, g_{n}+\theta\right)$. From Lemma 3.9, there is an $M>0$ large enough such that $h^{-1}(0)=\{0\}$ and $\operatorname{ind}_{0}(g)=\operatorname{ind}_{0}(h)$. Following a similar argument to the proof of Theorem 2.8, we can choose $M$ so that $\ell\left(v, g_{i}\right)=\ell\left(g_{i}+\theta\right)$ for all $v \in\left(\mathbb{R}_{+} \backslash\{0\}\right)^{n}$ and all $i=1, \ldots, n$, and we can prove that $h$ is also non-degenerate. Since $\Gamma_{+}\left(g_{i}+\theta\right)$ intersects each coordinate axis for $i=1, \ldots, n$, we can apply Corollary 4.7 to the map $h$.

We observe that the condition $\operatorname{supp}\left(G_{i}\right) \subseteq \Gamma_{+}\left(g_{i}\right) \backslash \Gamma\left(g_{i}\right)$ for all $i=$ $1, \ldots, n$ is equivalent to saying that for all $i=1, \ldots, n$ and all $v \in \mathbb{R}_{+}^{n} \backslash\{0\}$, the following condition holds:

$$
\begin{cases}\ell\left(v, G_{i}\right)>\ell\left(v, g_{i}\right) & \text { if } v \in\left(\mathbb{R}_{+} \backslash\{0\}\right)^{n}, \\ \ell\left(v, G_{i}\right) \geq \ell\left(v, g_{i}\right) & \text { otherwise. }\end{cases}
$$

In particular, $\ell\left(v, G_{i}\right)>\ell\left(v, g_{i}+\theta\right)$ for all $v \in \mathcal{F}_{\mathrm{c}}\left(\Gamma_{+}\left(g_{1}+\theta\right)+\ldots+\Gamma_{+}\left(g_{n}+\theta\right)\right)$ and all $i=1, \ldots, n$. Therefore, the result follows by applying Corollary 4.7 to the map $h=\left(g_{1}+\theta, \ldots, g_{n}+\theta\right)$.

REMARK 4.9. If $g=\left(g_{1}, g_{2}\right):\left(\mathbb{R}^{2}, 0\right) \rightarrow\left(\mathbb{R}^{2}, 0\right)$ is a non-degenerate analytic map germ such that $g^{-1}(0)=\{0\}$, then $g$ is automatically strongly non-degenerate. This is because if $g^{-1}(0)=\{0\}$, then $\operatorname{supp}\left(g_{1}\right) \cup \operatorname{supp}\left(g_{2}\right)$ contains a pure monomial $x_{i}^{k_{i}}$ of each variable $x_{i}, i=1,2$. Therefore, Corollary 4.8 generalizes the result of [13] on the index of planar vector fields. 
Let $f \in \mathcal{A}_{n}$, say $f=\sum_{k} a_{k} x^{k}$. Then we define the principal part of $f$ as the polynomial given by the sum of those $a_{k} x^{k}$ such that $k \in \Gamma(f)$. We denote this polynomial by $p(f)$. Given an analytic map $f:\left(\mathbb{R}^{n}, 0\right) \rightarrow\left(\mathbb{R}^{n}, 0\right)$, $f=\left(f_{1}, \ldots, f_{n}\right)$, the principal part of $f$ is the map $p(f):\left(\mathbb{R}^{n}, 0\right) \rightarrow\left(\mathbb{R}^{n}, 0\right)$ given by $p(f)=\left(p\left(f_{1}\right), \ldots, p\left(f_{n}\right)\right)$. We observe that $f$ is non-degenerate if and only if $p(f)$ is non-degenerate. Moreover, Corollary 4.8 implies that if $f:\left(\mathbb{R}^{n}, 0\right) \rightarrow\left(\mathbb{R}^{n}, 0\right)$ is an analytic strongly non-degenerate map such that $p(f)^{-1}(0)=\{0\}$, then $f^{-1}(0)=\{0\}$ and $\operatorname{ind}_{0}(f)=\operatorname{ind}_{0}(p(f))$. This is also proved, for $n=2$, in the paper [13] of Gutiérrez-Ruas. As we shall see, Corollary 4.8 can be substantially improved in the case $n=2$. First, we give a preliminary definition. If $g=\left(g_{1}, g_{2}\right):\left(\mathbb{R}^{2}, 0\right) \rightarrow\left(\mathbb{R}^{2}, 0\right)$ is an analytic map germ such that $\mathcal{F}_{\mathrm{c}}\left(g_{1}\right) \neq \emptyset$ or $\mathcal{F}_{\mathrm{c}}\left(g_{2}\right) \neq \emptyset$, then we define $\Gamma_{+}^{g}\left(g_{i}\right)=\left\{k \in \mathbb{R}_{+}^{2}:\langle k, v\rangle \geq \ell\left(v, g_{i}\right)\right.$ for all $\left.v \in \mathcal{F}_{\mathrm{c}}\left(g_{1}\right) \cup \mathcal{F}_{\mathrm{c}}\left(g_{2}\right)\right\}, \quad i=1,2$. It is clear that $\Gamma_{+}^{g}\left(g_{i}\right)$ is also a Newton polyhedron; we denote by $\Gamma^{g}\left(g_{i}\right)$ the union of its compact faces. Moreover, it is obvious that $\Gamma_{+}\left(g_{i}\right) \subseteq \Gamma_{+}^{g}\left(g_{i}\right)$, with equality if $\Gamma_{+}\left(g_{i}\right)$ intersects each coordinate axis.

Corollary 4.10. Let $g=\left(g_{1}, g_{2}\right):\left(\mathbb{R}^{2}, 0\right) \rightarrow\left(\mathbb{R}^{2}, 0\right)$ be an analytic non-degenerate map germ such that $g^{-1}(0)=\{0\}$. Suppose that $\mathcal{F}_{\mathrm{c}}\left(g_{1}\right) \neq \emptyset$ or $\mathcal{F}_{\mathrm{c}}\left(g_{2}\right) \neq \emptyset$. Let $G=\left(G_{1}, G_{2}\right):\left(\mathbb{R}^{2}, 0\right) \rightarrow\left(\mathbb{R}^{2}, 0\right)$ be another analytic map germ such that $\operatorname{supp}\left(G_{i}\right) \subseteq \Gamma_{+}^{g}\left(g_{i}\right) \backslash \Gamma^{g}\left(g_{i}\right), i=1,2$. Then $(g+G)^{-1}(0)=\{0\}$ and $\operatorname{ind}_{0}(g+G)=\operatorname{ind}_{0}(g)$.

Proof. Since $g$ is non-degenerate, it is adapted to the Newton polyhedron $\Gamma_{+}=\Gamma_{+}\left(g_{1}\right)+\Gamma_{+}\left(g_{2}\right)$, by Proposition 4.5. Moreover, since $\Gamma_{+}\left(g_{1}\right) \cup \Gamma_{+}\left(g_{2}\right)$ meets each coordinate axis, $g$ is strongly adapted to the Newton polyhedron

$$
\Gamma_{+}^{g}=\left\{k \in \mathbb{R}_{+}^{2}:\langle k, v\rangle \geq \ell\left(v, \Gamma_{+}\right) \text {for all } v \in \mathcal{F}_{\mathrm{c}}\left(\Gamma_{+}\right)\right\} .
$$

It is obvious that $\mathcal{F}_{\mathrm{c}}\left(\Gamma_{+}\right)=\mathcal{F}_{\mathrm{c}}\left(\Gamma_{+}^{g}\right)$. Moreover $\mathcal{F}_{\mathrm{c}}\left(\Gamma_{+}\right)=\mathcal{F}_{\mathrm{c}}\left(g_{1}\right) \cup \mathcal{F}_{\mathrm{c}}\left(g_{2}\right)$, by Theorem 4.1 of [10]. Thus, the condition $\operatorname{supp}\left(G_{i}\right) \subseteq \Gamma_{+}^{g}\left(g_{i}\right), i=1,2$, means that $\ell\left(v, G_{i}\right)>\ell\left(v, g_{i}\right)$ for all $v \in \mathcal{F}_{\mathrm{c}}\left(\Gamma_{+}^{g}\right)$. But this implies the desired result as a consequence of Theorem 2.8 .

To finish the paper, we indicate how to get an upper bound for the minimum of those integers $r$ such that $\left(j^{r} g\right)^{-1}(0)=\{0\}$ and $\operatorname{ind}_{0}\left(j^{r} g\right)=$ $\operatorname{ind}_{0}(g)$, where $g:\left(\mathbb{R}^{n}, 0\right) \rightarrow\left(\mathbb{R}^{n}, 0\right)$ is any analytic function germ such that $g^{-1}(0)=\{0\}$ (see Lemma 3.9).

Let $g_{\mathbb{C}}:\left(\mathbb{C}^{n}, 0\right) \rightarrow\left(\mathbb{C}^{n}, 0\right)$ be the function germ obtained from $g$ by complexifying the variables $x_{1}, \ldots, x_{n}$. Suppose that $g_{\mathbb{C}}$ converges in some neighbourhood of the origin in $\mathbb{C}^{n}$. Let $I\left(g_{\mathbb{C}}\right)$ denote the ideal of $\mathcal{O}_{n}$ generated by the components of $g_{\mathbb{C}}$; suppose that $V\left(I\left(g_{\mathbb{C}}\right)\right)=\{0\}$. Thus, by the Nullstellensatz [17, p. 196], we can consider the number

$$
r_{0}\left(g_{\mathbb{C}}\right)=\min \left\{r \geq 1: m_{n, \mathbb{C}}^{r} \subseteq \overline{I\left(g_{\mathbb{C}}\right)}\right\},
$$


where $\overline{I\left(g_{\mathbb{C}}\right)}$ stands for the integral closure of $I\left(g_{\mathbb{C}}\right)$ in $\mathcal{O}_{n}$ and $m_{n, \mathbb{C}}$ is the maximal ideal of $\mathcal{O}_{n}$. If we define

$$
r_{0}(g)=\min \left\{r \geq 1: m_{n}^{r} \subseteq \overline{I(g)}\right\},
$$

where $\overline{I(g)}$ denotes the integral closure of $I(g)$ in $\mathcal{A}_{n}$, then $r_{0}(g) \leq r_{0}\left(g_{\mathbb{C}}\right)$, by Definition 3.1. The number $r_{0}(g)$ is the minimum integer greater than or equal to the Eojasiewicz exponent $\alpha_{0}(g)$. Note that if $g^{-1}(0)=\{0\}$, then $\alpha_{0}(g)$ always exists, by Theorem 3.2, hence $r_{0}(g)$ cannot be $\infty$ in this case, although $r_{0}\left(g_{\mathbb{C}}\right)$ may be $\infty$ if we do not assume that $V\left(I\left(g_{\mathbb{C}}\right)\right)=\{0\}$. In [1], the author shows an algorithm to compute $r_{0}\left(g_{\mathbb{C}}\right)$, assuming that $V\left(I\left(g_{\mathbb{C}}\right)\right)$ $=\{0\}$, through the program Singular [11] (the number $r_{0}\left(g_{\mathbb{C}}\right)$ is related to the notion of topological sufficiency of jets). We observe, by Remark 3.10, that $\left(j^{r} g\right)^{-1}(0)=\{0\}$ and $\operatorname{ind}_{0}(g)=\operatorname{ind}_{0}\left(j^{r} g\right)$ for all $r \geq r_{0}\left(g_{\mathbb{C}}\right)$.

EXAMPLE 4.11. Consider the analytic map $g:\left(\mathbb{R}^{2}, 0\right) \rightarrow\left(\mathbb{R}^{2}, 0\right)$ given by $g(x, y)=\left(x^{9}+y^{9}+x^{2} y^{5}-x^{5} y^{2}, x y^{3}-x^{3} y\right)$. We observe that $g$ is a degenerate map germ and that $V\left(I\left(g_{\mathbb{C}}\right)\right)=\{0\}$. Applying the algorithm of $[1]$, we find that $r_{0}\left(g_{\mathbb{C}}\right)=9$. Thus, any analytic map $f:\left(\mathbb{R}^{2}, 0\right) \rightarrow\left(\mathbb{R}^{2}, 0\right)$ such that $j^{9} f=j^{9} g$ satisfies $f^{-1}(0)=\{0\}$ and $\operatorname{ind}_{0}(g)=\operatorname{ind}_{0}(f)$.

ExAmple 4.12. Let $g:\left(\mathbb{R}^{2}, 0\right) \rightarrow\left(\mathbb{R}^{2}, 0\right)$ be given by $g(x, y)=\left(x^{3}+\right.$ $\left.x y^{2}, x^{2} y+y^{3}\right)$. Since $g^{-1}(0)=\{0\}$, the index of $g$ is defined, and $\operatorname{ind}_{0}(g)=1$. The map $g$ is non-degenerate, and therefore strongly non-degenerate (see Remark 4.9). As a consequence, if we consider any analytic map germ $G=\left(G_{1}, G_{2}\right):\left(\mathbb{R}^{2}, 0\right) \rightarrow\left(\mathbb{R}^{2}, 0\right)$ such that $k_{1}+k_{2}>3$ for all $\left(k_{1}, k_{2}\right) \in$ $\operatorname{supp}\left(G_{1}\right) \cup \operatorname{supp}\left(G_{2}\right)$, then $(g+G)^{-1}(0)=\{0\}$ and $\operatorname{ind}_{0}(g+G)=\operatorname{ind}_{0}(g)$, by Corollary 4.10. This can also be concluded from Theorem 1.1, since $g$ is a homogeneous map.

We also observe that the ideal $I\left(g_{\mathbb{C}}\right)$ of $\mathcal{O}_{2}$ does not have finite codimension, that is, $V\left(I\left(g_{\mathbb{C}}\right)\right) \neq\{0\}$. Thus $r_{0}\left(g_{\mathbb{C}}\right)=\infty$ but $r_{0}(g)=3$, by Theorem 3.5.

Acknowledgements. The author wishes to express his gratitude to Prof. M. A. S. Ruas for her helpful comments and to the members of the Department of Mathematics of the ICMC of the University of São Paulo at São Carlos (Brazil) for their hospitality.

\section{References}

[1] C. Bivià-Ausina, A method to estimate the degree of $C^{0}$-sufficiency of analytic functions, Experiment. Math. 11 (2002), 81-85.

[2] - Eojasiewicz exponents, the integral closure of ideals and Newton polyhedrons, J. Math. Soc. Japan (2003), to appear. 
[3] C. Bivià-Ausina, T. Fukui and M. J. Saia, Newton filtrations, graded algebras and codimension of non-degenerate ideals, Math. Proc. Cambridge Philos. Soc. 133 (2002), 55-75.

[4] J. Bochnak and J. J. Risler, Sur les exposants de Eojasiewicz, Comment. Math. Helv. 50 (1975), 493-507.

[5] M. Brunella and M. Miari, Topological equivalence of a plane vector field with its principal part defined through Newton polyhedra, J. Differential Equations 85 (1990), $338-366$.

[6] A. Cima, A. Gasull and J. Torregrosa, On the relation between index and multiplicity, J. London Math. Soc. (2) 57 (1998), 757-768.

[7] D. Eisenbud and H. I. Levine, An algebraic formula for the degree of a $C^{\infty}$ map germ, Ann. of Math. 106 (1977), 19-44.

[8] T. Fukui, The modified analytic trivialization of a family of real analytic mappings, in: Contemp. Math. 90, Amer. Math. Soc., 1989, 73-89.

[9] T. Gaffney, Integral closure of modules and Whitney equisingularity, Invent. Math. 107 (1992), 301-322.

[10] J. F. Gately, Unique factorization of *-products of one-fibered monomial ideals, Comm. Algebra 28 (2000), 3137-3153.

[11] G. M. Greuel, G. Pfister and H. Schönemann, Singular version 1.2. User manual, Reports On Computer Algebra 21, Centre for Computer Algebra, Univ. of Kaiserslautern, 1998. http://www.mathematik.uni-kl.de/zca/Singular

[12] V. Guillemin and A. Pollack, Differential Topology, Prentice-Hall, Englewood Cliffs, NJ, 1974.

[13] C. Gutiérrez and M. A. S. Ruas, Indices of Newton non-degenerate vector fields and a conjecture of Loewner for surfaces in $\mathbb{R}^{4}$, preprint, 2001.

[14] A. G. Khovanskiı̌, Newton polyhedra and toroidal varieties, Funct. Anal. Appl. 11 (1977), 289-295.

[15] M. Lejeune et B. Teissier, Clôture intégrale des idéaux et equisingularité, Centre de Mathématiques, Univ. Scientifique et Medicale de Grenoble, 1974.

[16] S. Łojasiewicz, Ensembles semi-analytiques, IHES, 1965.

[17] —, Introduction to Complex Analytic Geometry, Birkhäuser, 1991.

[18] J. Milnor, Topology from the Differentiable Viewpoint, Univ. Press of Virginia, 1965.

[19] J. J. Risler, Les exposants de Lojasiewicz dans le cas analytique réel, appendix to $[15]$.

[20] B. Teissier, Cycles évanescents, sections planes et conditions de Whitney, in: Singularités à Cargèse (Cargèse, 1972), Astérisque 7-8 (1973), 285-362.

[21] V. Županović, Topological equivalence of planar vector fields and their generalised principal part, J. Differential Equations 167 (2000), 1-15.

Departament de Matemàtica Aplicada

Universitat Politècnica de València

Escola Politècnica Superior d'Alcoi

Plaça Ferrándiz i Carbonell 2

03801 Alcoi (Alacant), Spain

E-mail: carbivia@mat.upv.es

Received 3 July 2002;

in revised form 12 March 2003 